\title{
What is a target system?
}

\author{
Alkistis Elliott-Graves ${ }^{1}$
}

Received: 5 July 2018 / Accepted: 4 March 2020 / Published online: 12 March 2020

(c) The Author(s) 2020

\begin{abstract}
Many phenomena in the natural world are complex, so scientists study them through simplified and idealised models. Philosophers of science have sought to explain how these models relate to the world. On most accounts, models do not represent the world directly, but through target systems. However, our knowledge of target systems is incomplete. First, what is the process by which target systems come about? Second, what types of entity are they? I argue that the basic conception of target systems, on which other conceptions depend, is as parts of the world. I outline the process of target system specification and show that it is a crucial step in modelling. I also develop an account of target system evaluation, based on aptness. Paying close attention to target system specification and evaluation can help scientists minimise the frequency and extent of mistakes, when they are using models to investigate phenomena in complex real-world systems.
\end{abstract}

Keywords Target system $\cdot$ Model-world relations $\cdot$ Relevance $\cdot$ Omission $\cdot$ Ecology

\section{Introduction}

The marmots of Vancouver Island (Marmota vancouverensis) are classified as critically endangered. It was estimated that their population dropped $80-90 \%$ since the 1980s and reached a low of 70 individuals in 1998 (Brashares et al. 2010). Yet the cause of this rapid decline was a mystery. The marmots were not hunted, their sources of food were unaltered, there were no new predators or competitors and the small disturbances to their habitat (small-scale logging) seemed to have a positive effect on the population, as the absence of thick tree roots in clearings made the building of burrows much easier. Nonetheless, the marmot population began to decline in the 1980s and kept on declining, despite some of the early conservation

Alkistis Elliott-Graves

alkistis.elliott-graves@helsinki.fi

1 Department of Practical Philosophy, Center for Philosophy of Social Science (TINT), University of Helsinki, Unioninkatu 40A, P.O. Box 24, 00014 Helsinki, Finland 
efforts (such as the expansion of Strathcona Provincial Park in 1995). Something had to be done quickly to preserve the species from extinction.

As is often the case when dealing with highly complex systems, the scientists who took on the problem of the Vancouver Island Marmots (VIM) adopted a modelling approach. They used a simple and highly idealised model: logistic growth. This model measures how the growth rate of a population is limited by the density of the population itself, that is it describes how populations grow when they have limited environmental resources and are not subject to other limiting factors such as competition with other species, predation, migration etc. But how did the scientists decide which model to use? What was the connection between the model and the actual marmots?

There is substantial literature that addressing how idealised models can provide information about real world phenomena. On many of these accounts, models do not represent the world directly, but target some aspects of the world and represent those (Knuuttila 2005; Matthewson and Weisberg 2009; Suárez 2010; Peschard 2010). These target systems can be many types of things, such as other models, hypothetical systems, images, equations (see for example Suarez 2003; Weisberg 2013), though the consensus seems to be that in the simplest cases of model-world relations, target systems are parts of the world (Frigg 2009; Giere 2004; Peschard 2010). Thus, the short answer to the above question is that if models represent target systems and target systems are parts of the world, then models provide information about the world.

Even though this picture is largely correct, there are many interesting questions lurking beneath the surface. The most important of these are: 'which parts of the world?' and 'how are they determined?' So far, the most coherent answers to these questions have been given by the somewhat complementary accounts of Peschard (2010) and Weisberg (2013). According to Peschard, modellers target the relevant parts of the world for the phenomenon they are studying. According to Weisberg, target systems are determined through a process of abstraction. In other words, modellers determine a target system by deciding which aspects of the phenomenon of interest is relevant for their model. I agree with Weisberg and Peshcard that this is part of determining a target system, but it is not the full story. In what follows, I will argue that it is only one of four steps in target system specification.

Moreover, though these accounts of model-world relations do more than previous ones to highlight the existence of target systems, they still do not fully appreciate the importance and role of target systems in scientific practice. For example, Weisberg presents the determination of target systems as a simple and straightforward matter, or at least as a scientific matter to be determined on a case-by-case basis, not of philosophical interest (p. 92). He dismisses any difficulties in determining target systems as the hallmark of young disciplines. As the disciplines mature, the target systems solidify and if disagreement continues, this "may ultimately split a research community into subfields which cannot see eye to eye" (p. 92).

Weisberg is right to claim that disagreement regarding target systems can lead to splitting a research community. A discipline that fits this picture is Ecology. Traditionally, Ecology was seen to have progressed from a merely observational to a truly scientific field with the introduction of mathematical models in the late nineteenth Century (Shrader-Frechette and McCoy 1993). These were population-level models with few parameters, that were meant to apply widely 
to populations across different taxa (Kingsland 1995). Yet these simple models did not have sufficiently high predictive success, so ecologists began searching for alternative ways to approach problems. The main differences in these alternative approaches were their different conceptions of ecological systems, such as in terms of entire communities or ecosystems, rather than individual populations (Odenbaugh 2007). Nowadays, the different subfields within ecology do not, as Weisberg states, see 'eye to eye' regarding the appropriate level of grain at which ecological systems should be studied, and consequently regarding the appropriate type of model that should be used to investigate various ecological phenomena.

However, this should be very interesting to philosophers. Why think, for instance, that ecosystem-sized target is better for understanding ecological phenomena than a target system comprised of populations? Moreover, are target systems truly determined within each subfield? For instance, community ecology is one of these recognised subfields, yet there are disagreements within the field between those who employ a holistic (following Clements) and those who adopt an individualistic (following Gleason) conception of a community (Odenbaugh 2007). Finally, even if scientists have determined a particular approach within a subfield, is their target system fully determined? For example, the scientists studying the VIM were working within the discipline of population ecology. Can we infer from this what their target system was? I do not believe that we can. In what follows, I will argue that determining the appropriate target system for a particular scientific investigation is laborious, time-consuming and fraught with difficulties even for scientists working within an established discipline or sub-discipline. In fact, Ecology is a particularly apt discipline to focus on for the explication of the use and role of target systems. This is because, on the one hand, some scientists (such as Brashares et al.) provide meticulous detail of their targets, but on the other hand, there are many examples within the discipline, where failure to correctly specify targets resulted in inaccurate model predictions. Thus, understanding the use and role of target systems in Ecology is a very good place to begin a philosophical investigation of target systems in science.

The paper is structured in the following way. In section "Specifying a target system", I lay out the basics of my view on target systems. I start by examining the VIM case in detail, highlighting four processes that were necessary for preparing the 'world' so that the model could be applied to it (2.1). I then provide a more general account of target system specification (2.2), followed by the explication of the ontological status of target systems (2.3), and an examination of when a full-blown investigation of target systems is likely to be fruitful (2.4). I then turn to how target systems can be evaluated, with the notion of aptness, and examine two different types of mistakes that can be made in the course of target system specification, which can have far-reaching consequences for actual scientific practice ("Evaluating target systems"). In section "Loose ends: general and hypothetical targets", I examine more complex cases, where the targets of models are not straightforward parts of the world but generalised or hypothetical systems. I argue that genuine cases of general and hypothetical targets are actually infrequent in science, but that we should still adopt an overall pluralistic attitude towards the nature of target systems. 
a Per capita Logistic Growth Curve and Allee efects

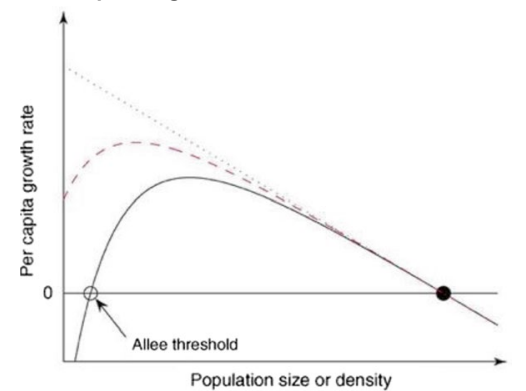

The relationships between the per capita population growth rate and either population size or dens ity for negative density dependence (dotted curve), and weak (dashed curve) and strong (solid curve) Allee ef fects. In weak or strong Allee ef fects, the relationship is positive at $\mathrm{l}$ ow population sizes or densities, where positive densitydependent (Allee effect ) mechanisms overpowe $r$ negative density-dependent (intraspecific competition) ones. It is negative at high population sizes or densities, where the converse is true. Reprinted from (Berec et al. 2007) b Allee efect in the VIM

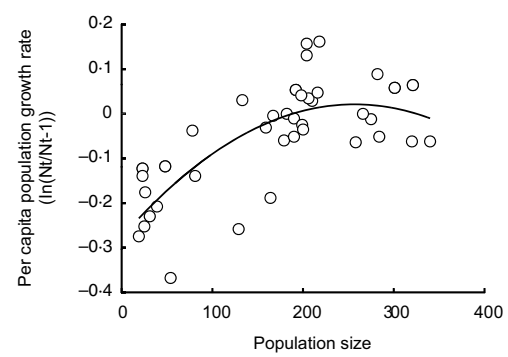

Annual c ounts of free-living V. I. ma rmots reveal strong i nverse density dependence in per capita population growth from 1970 to 2007. Trend line represents least-squares quadratic fit $\left(\mathrm{R}^{2}=0 Æ 55\right)$. Data e xclude a nimals introduced from captivity. (Reprinted from Brashares et al. 2010)

Fig. 1 Logistic growth and the Allee effect

\section{Specifying a target system}

\section{The VIM target system}

Let us start by examining the case of the VIM in more detail. How did the scientists go about investigating VIM growth? They realised that the VIM population was not described by the standard logistic growth equation (whose differential form can be seen in Eq. 1, see also Fig. 1a dotted line). Normally, as an environment becomes more densely populated, the growth rate of the population drops, as there are not enough resources to sustain an exponentially growing population.

$$
\frac{d N}{d t}=r N\left(1-\frac{N}{K}\right)
$$

Here, $(r)$ is the intrinsic growth rate, the maximum possible growth rate of the population. The second important component of the model is $(K)$, the carrying capacity of the environment. $(K)$ imposes the upper limit on population growth because it is the maximum number of individual organisms that a particular environment can support. $(K)$ is determined by the availability of resources in the environment and varies across environments and species. Populations displaying logistic growth have an initial phase of rapid growth, where the growth rate itself increases exponentially. As resources are used up, the growth rate slows, until the carrying capacity is reached, whereupon the growth rate stabilises.

The problem was that the VIM's population was dropping even though there seemed to be abundant resources. The scientists hypothesized that the cause of the VIM population decline was due to an 'Allee effect', which occurs when there is a positive correlation between the density of a population and its growth rate at low population densities (Fig. 1a, red and black curves). This means that when a 
Fig. 2 The domain of studygeographical location of VIM populations

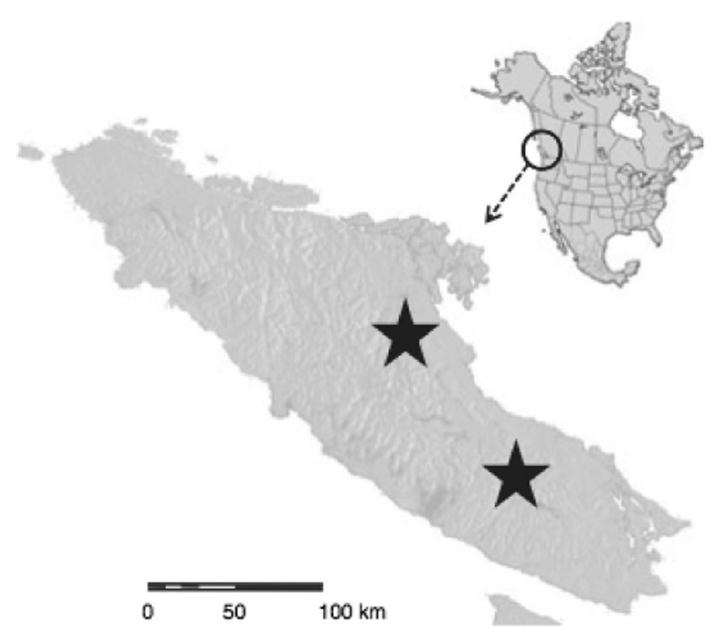

population size drops to a sufficiently low density, it will not recover despite an abundance of resources (Courchamp et al. 1999).

Figure $1 \mathrm{~b}$ shows that the VIM were in fact exhibiting an Allee effect. The scientific investigation revealed that this was caused by a peculiarity of the VIM, namely that they are highly social. When the population falls below a certain density, the VIM find it hard to locate potential mates, especially because the smaller populations mean that there is less division of labour (caring for young, guarding against predators) so each marmot spends more time and effort foraging and looking for mates. All this results in lower instances of mating, hence the drop in population despite the abundance of resources.

Yet even if scientists have a hypothesis about what model to use for a particular investigation, how do they apply the model to the world? More specifically, what exactly do they apply the model to? In other words, what needs to happen so that we get from Fig. 1a, b? Merely stating that they applied the model to the VIM, does not do justice to the time and effort spent 'preparing' the 'world' so that the model could be applied to it. As it happens, Brashares and colleagues documented this process in some detail. In fact, these scientists are part of a small but steadily growing group of researchers who are beginning to acknowledge the importance of this preliminary work and publish it, albeit in the paper's supplementary materials (reprinted here in Box 1). It is this preliminary work that corresponds to the process of target system specification.

Specifying a target system is a four-part process. The first step was to determine exactly where the study would take place, i.e. the spatio-temporal location where the phenomenon manifests. I call this the domain of study. Here, the domain of study encompasses the two areas in Vancouver Island with VIM populations (Fig. 2).

I should note that identifying the domain of study is sometimes rather obvious, and can be inferred from the context of the paper, so it is not always explicitly stated. However, in some cases, such as this one, the extent of the domain is simply not obvious at first glance, so it is useful to delineate the exact domain of the 
phenomenon under investigation. In order to do this, the scientists had to estimate the territories of the marmot populations and make sure that the two populations did not overlap. This is conceptually straightforward but quite laborious, as it is based on actual long-term observations of a subset of marmots (see Box 1A for a graphical representation of the territory estimate). This information was also useful because it demonstrated that the populations had not been displaced in the last 75 years. This, in turn, showed that the spatial variables of the VIM remained unchanged, so the scientists could legitimately avail themselves of pre-existing data on the VIM populations, dating back to 1973 .

The second step was to partition the domain. Put simply, partitioning is the division of the domain of study into parts. ${ }^{1}$ In other words, when partitioning, a scientist decides how to carve up the domain of study. This often amounts to deciding the level of grain at which the domain will be partitioned. For example, the VIM domain was partitioned at the level of organisms (e.g. individual marmots, plants, insects etc.). An alternative partition of the same domain could be at the level of meta-populations or whole ecosystems, if, for instance, the scientists approached the phenomenon from an ecosystem ecology perspective. ${ }^{2}$ Partitioning also involves identifying properties that correspond to each part. Each part, e.g. an individual marmot, will have a long list of properties that pertain to it; it will have a certain weight and height, a certain age, it will be male or female, its fur will be a certain shade of brown, its eyes will be a certain colour and so on.

The third step is to determine which parts and properties are relevant for the investigation. Here, the marmots themselves and at least some of their demographic properties (e.g. being male or female, being a certain age, having a particular gestation period) are obviously relevant, because they are necessary for investigating the growth of a population (whichever growth model is used). For example, the procedure for determining $r$ (the intrinsic growth rate for the logistic growth model) is as follows: first, the scientists collect data on birth and death events and on the age, sex and gestation period of the population. These are used to construct an age-structured 'life table', a tabular summary of the birth rates, death rates and fecundity of the population (age, sex and gestation period are used to calculate fecundity). The values in the table are then used to calculate $r$ (Ricklefs and Miller 2000) (See Box 1B for a visual representation of a part of this process).

Still, knowing that a property is relevant does not mean that it can be used. Sometimes, the data is simply not available, or not of sufficiently high quality. Even something as conceptually straightforward as determining the age structure

\footnotetext{
1 I will be using the terms 'parts' and 'properties' throughout this paper. By parts, I mean parts of the world. I use this term because I want to emphasize that these units have the same ontological status as the larger system which they find themselves in. The natural system is the whole, and the units are some parts of it. The parts have not been altered or changed in any way. I use the term 'properties' simply to refer to characteristics or features of these units. A detailed account of the nature of properties is beyond the scope of this paper. Moreover, the nature of this analysis is such that it can remain silent on the fundamental nature of properties. All that is needed is a way to refer to features that are predicated or instantiated, universally or not, by the units of the analysis.

2 I will return to the topic of alternative partitions in sections "The ontological status of target systems" and "Loose ends: general and hypothetical targets".
} 
and sex ratio of a population is difficult, as it is based on observations of a sample of the population. Furthermore, even with the best technology (i.e. tags), the data can be very patchy. In this case, the scientists had to spend three years observing the marmots and documenting all the properties they deemed relevant. Then, they had to compare their observational data with the existing observational data (from the 70s), in order to be sure that data from both periods could be used (see Box 1B).

Some aspects of the system are obviously irrelevant, such as marmot eye colour or the gravitational force of the moon. Other factors are not obviously irrelevant, but can 'safely ignored'. In some cases, the scientists might not know that a factor is irrelevant, but can make an educated guess, based on existing ecological knowledge. For example, factors such as temperature, might have an effect on marmot growth, but if the temperature in the last five years falls within the appropriate range, then the scientists can assume that has not been the cause of the decline.

Other aspects might require closer scrutiny. For example, an increase in the number of predators could be the cause of the decline. Scientists know that the VIM are preyed on by wolves, eagles and cougars. They also knew that there had been a very small increase in the predation rate in the mid-1990s. However, this increase was too small to account for the magnitude of the drop in the marmot population. Second, such an increase in predation rate is usually accompanied by a corresponding rise in reproductive rate of the prey population. In this case, however, the marmot reproduction was falling and at an increasing rate! Thus, the scientists decided to omit predation from their investigation and focus instead on the factors that affected the reproductive rate of the VIM.

At this point, the scientists have specified a target system. They have decided the grain at which the domain will be partitioned (i.e. individual marmots rather than meta-populations or marmot alleles), they have decided which properties of the marmots are relevant (demographic properties) and they have also decided that no other organisms are relevant for the investigation (e.g. particular prey, predators or competitors). As it turns out, this was also a successful case of target system specification. The scientists chose an apt target system that allowed them to determine that the standard logistic growth model did apply sufficiently well to the population, and were then able to determine that there was something missing from their target system. Moreover, what was missing was not one of the most common factors, such as competition or predation, but a factor entirely specific to the VIM, whose importance was hitherto unknown (sociality). Still, because the scientists had conducted the preliminary work, they were able to recognise that there were differences in the time allocated to foraging versus socialising between the 1970s and 2000s populations (see Box $1 \mathrm{D}$ ). This information was incorporated into the growth model (with the Allee effect), which then described the population's growth much more accurately. I should note that the sociality-induced Allee effect from this study has since been incorporated into the highly successful VIM recovery programme, which focuses predominantly on increasing the VIM population through captive breeding (as opposed to the most common alternative strategy: culling of predators) (Vancouver Island Marmot Recovery Team 2008). 


\section{Specifying a target system: a more general account}

The VIM case was relatively simple and straightforward. The scientists were investigating a well-known phenomenon in a clearly defined domain. Moreover, though their choice of partition and omissions were time-consuming and laborious, it was conceptually straightforward: their partition was typical for population ecology, there were few factors whose relevance needed to be checked, and most importantly, there were no factors that were mistakenly omitted as irrelevant. However, specifying a target system is often much more difficult. In this section, I will give a more general account of target system specification indicating where, how and why difficulties are likely to manifest.

The motivation for specifying a target system is that a model cannot be applied to a system without some preparation. Scientists must pick out the parts and properties of a real-world system that they wish to study, given the context of a particular phenomenon. The first point to note is that on my view, there is a distinction between a target system and a phenomenon. This is important because sometimes philosophers use the two terms interchangeably (see for example Matthewson and Weisberg 2009, p. 180). Here, phenomena are states or behaviours of a real-world system or group of systems, studied by a particular discipline, such as population growth, competition or predation. A target system is those aspects of the real-world system that are studied in order to gain knowledge about the phenomenon.

Moving on to the process itself, the domain of study is where the phenomenon takes place. In simple cases, this amounts to a spatio-temporal region such as an ecosystem, the Pacific Ocean, a cylinder containing gas, or in the social sciences, a setting which counts as the environment for a group of people, such as a neighbourhood, a church or a laboratory. There are more complex cases where the system being studied does not exist in the world. These will be discussed in section "Targets of hypothetical models".

Partitioning is where things start to get really interesting. In some cases, partitions are standardised within disciplines. As we saw, in population ecology the standard partitions are individual organisms. Other disciplines have different standardised partitions: in population genetics, domains are partitioned at the level of alleles, in organic chemistry partitions are at the grain of organic molecules, in physics they may be particles, in psychology they are individuals, in anthropology they are groups of individuals and so on.

So far, I have described simple cases where the scientist partitions the domain into roughly equally sized parts. However, it is also possible for partitions to cut across levels of specificity. For example, a plant ecologist studying competition, might group an individual plant and the mycorrhizal fungi in its roots as one part, and the nitrogen which the fungi help the plant to absorb as another part. Alternatively, she might partition the plants into individual parts and group together the entire population of fungi with the nitrogen molecules as 'below-ground factors'. ${ }^{3}$

Once the partition is set, scientists decide which parts and properties defined by the partition are relevant and which are not. Those that are not considered relevant

\footnotetext{
${ }^{3}$ For examples of such a partitions, see (Phillips et al. 2016; Sackett et al. 2010).
} 
are omitted. Omission is best understood as ignoring certain aspects of a system. It works in the same way as putting a coloured plastic film over a picture; the objects that are the same colour as the film disappear. For example, if we put a green film over a picture of vase of red roses on a blue background, then the leaves and stems of the roses will disappear. If we put a red film over the picture, then the rosebuds will disappear. The ability to choose between different film colours is analogous to the scientists' ability to choose between different methods or models to study a particular phenomenon. The parts and properties that do not show up through a particular filter are analogous to the omitted factors, while the remaining parts of the picture that are still visible are the analogues of the parts and properties that make up the target system.

Two clarifications. First, are partitioning and omission truly distinct? Doesn't partitioning (especially in the case of cross-cutting partitions) implicitly include omission? Conversely, doesn't a particular omission imply a particular partition? I agree that it may seem that the distinction is superfluous, especially since scientists do not actually distinguish between how they partition a domain and how they choose what to omit. Nonetheless, I believe that there is a conceptual distinction between the two processes, even if they are not always distinguishable in practice. Partitioning is carving the domain into parts. The same domain can be partitioned in many different ways, but all those partitions will contain the same amount of 'stuff'. The difference between partitions is that this 'stuff' is organised differently. Omission, on the other hand, is the assertion that some of this stuff is not relevant for the purposes at hand. Different parts and properties can be omitted from partitioned domain, depending on the purpose at hand.

Second, my notion of omission seems very similar to what others have termed 'abstraction'. For example, Weisberg defines target systems as abstractions over real phenomena, generated through a process of abstracting (2013 p. 116). I prefer the term 'omission', because 'abstraction' is quite ambiguous in philosophy, as it can refer to non-concrete concepts or ideas (Cartwright 1989), generalizations (Levy 2018), isolations (Mäki 2009) or parts of a larger system (Jones 2005), and whether or not all these terms are coextensive is a matter of debate (Godfrey-Smith 2009; Humphreys 1995; Levy 2018). Moreover, on some of these accounts, it is possible for an abstract product to come about through different processes (Levy 2018). In the case of target system specification, the process matters. Target systems are not specified by other 'abstracting' processes such as de-concretizing or generalizingthey are specified through partitioning and omission. Finally, my choice of terminology avoids some of the problems faced by Weisberg, when distinguishing between merely abstract and generalized target systems (see section "Loose ends: general and hypothetical targets").

\section{The ontological status of target systems}

I have outlined the process of target system specification, but what of the products yielded by this process? That is, what sorts of things are target systems? As stated in the introduction, they are usually thought of as parts of the world. But what exactly does this mean? What is the ontological status of a part of the world? The short 
answer is that it is concrete and therefore real. Yet a possibly worry is that the process of specifying a target system, which includes partitioning and omission, yields entities that are mere representations, rather than concrete and real parts of the world. The response is that there is a difference between referring to target systems and target systems themselves. When we talk or write about a target system, we are referring to it or representing it in a way similar to the way a model represents it. This representation can be simplified or distorted, yet the neither the existence of the representation nor the process of representing alters the system itself. The processes of partitioning and omission are equally non-intrusive with respect to the ontological status of the parts of the target system. All we do when we partition and identify relevant parts and properties, is group a part of the world in a particular way. But this does not change the parts themselves. The point is that the target system has the same ontological status as the domain. If we think that my laptop is concrete and real, then the ' $\mathrm{R}$ ' key on the keyboard also concrete and real. ${ }^{4}$

\section{When are target systems important?}

Now that the basics of target system specification are in place, we can think about their value. Do all scientists always specify targets? Should they? Are there some disciplines and/or types of investigation where targets are more valuable? The short answer is that many scientists do not need to spend time and effort specifying target systems. This is because there are some disciplines/sub-disciplines/types of scientific investigation where the target systems are too obvious to need particular attention. For example, if a scientist is interested in determining whether a particular substance will kill a bacterium, their target system is clear and straightforward. The experimental setup will exclude/control all other factors and the scientist will then determine the effect of the substance on the bacterium in this precise context. Of course, this does not mean that the scientific investigation does not have a target system, merely that it is not necessary to spend any time specifying it in detail.

In fact, the value of specifying target systems often becomes apparent only when scientists run into difficulties. This is quite common in investigations of complex systems, where scientists have yet to determine which parts of a network of interconnected causes are actually responsible for particular phenomena. This is why target systems are especially important in disciplines such as Ecology. In addition to the underlying complexity of ecological systems, there are deep disagreements in the field about the best concepts and methods that should be used to conduct ecological investigations.

A sceptical reader may point out that this is whole point of the investigating the phenomena in the first place. That is, isn't it enough to see whether a particular model correctly predicts the behaviour of the system? Specifying a target system in detail can help to avoid model failure or to deal with an inaccurate prediction.

\footnotetext{
${ }^{4}$ Of course, it is possible to insist that any partition is a mere convention. Yet if this is the case then the whole domain must also be a convention, because the domain is itself partitioned from the rest of the world. The point is that the ontological status of the target system is the same as that of the domain.
} 
For example, applying the basic logistic growth model to the VIM target system revealed that something was amiss, as the model did not accurately describe the population. Target system specification showed that an additional factor was relevant in this case, namely the VIM sociality. In this case, there was no need to change the model being used, as the same model could easily be modified to describe a population with an Allee effect, i.e. a population that is affected by density, but only above a certain threshold. However, in other cases, model failure or inaccurate predictions might not be easy to remedy. Sometimes the use of a particular type of model persists because scientists have not realised that the cause of predictive failure is the absence of a relevant factor from the model. An example of this comes from plant ecology, where for many years, scientists failed to solve the problem of erratic predictions in models of drought sensitivity, because of a persistence of 'surface bias' (Phillips et al. 2016). Even though scientists knew that below-ground factors affected plant growth, they mistakenly believed them to be irrelevant for their models. Only after re-examination of their targets did they realise the importance of these factors. ${ }^{5}$

Finally, target system specification can aid in model choice. Specifying the target system, or even comparing two or more target systems from a domain is an important part of the preliminary work for determining which model to use for a particular purpose. Again, this is not always straightforward. For example, there is a longstanding debate, in ecology, concerning the optimal level of model complexity. On the one hand, reducing complexity allows scientists to distinguish between the core causal factors that give rise to classes of phenomena and mere details that are idiosyncratic to particular systems (May 2001; Perretti and Munch 2013). On the other hand, mirroring the complexity of real-world systems increases the likelihood that models capture all the relevant causal factors and dynamics giving rise to complex phenomena (Evans et al. 2013; Travis et al. 2014). A close look at target systems can show when scientists should opt for more or less complexity in their models.

\section{Evaluating target systems}

It may seem rather odd to think that we can evaluate target systems, if they are just real parts of the world. It does not really make sense to think of a part of the world, such as a field or a flower or a desk as good or bad per se. Nonetheless, it does make sense to compare parts of the world, for a particular purpose and within a particular context. For example, one field may be better than another field for growing a particular crop, or better than a cliff for the purposes of playing volleyball. It can be worse than another field for the establishment of a biological pest and worse than a river for farming trout. Similarly, in the case of target systems, when we evaluate them, we are not trying to determine whether they are good or bad in themselves, but whether they are apt for a particular purpose. A full-blown account of target system aptness is beyond the scope of this paper, but for the remainder of this section I

\footnotetext{
$\overline{5}$ For more details on this example, see section "Evaluating target systems".
} 
will provide a general outline of the account, illustrated by some examples of inapt targets.

Recall that target system specification is comprised of two main elements, partitioning the domain into parts and properties and determining which of those parts and properties are relevant for the purposes of the scientific investigation. A preliminary definition of aptness is the following:

A target system $T$ is apt for a scientific purpose $S$ just in case:

(i) Its partition $P$ is useful for $S$ and

(ii) $\quad P^{*}$ (the partition after omission) contains all and only the relevant causal factors for $S$.

In order to make sense of this definition, we need to determine what is meant by useful and relevant. I will use aspects of the kairetic account of causal explanation, which provides a way of identifying the actual causes of an event from a wider web of potential factors (Strevens 2004, 2008). A few notes on this choice. There are many areas in philosophy where usefulness and relevance are examined, especially in philosophy of language. ${ }^{6}$ However, I have chosen the approach taken in by the literature on scientific explanation, specifically Strevens's account, for the following reasons. First, the type of 'context' investigated in scientific explanation is the same as in the case of target systems, and it is affected by scientific disciplines, norms and practices. While these may have analogues in communication and cognition, it is simpler to use the theory where context is restricted to scientific practice. Second, Strevens distinguishes between three types of causal factors: those that true difference makers, those that could have causal influence on an event but did not and those that are merely tangential. One of the important difficulties faced by scientists applying models to real world systems is to determine which out of a network of possible causes are true difference-makers. Thus, an account that makes this exact distinction ought to be helpful in an account of target system evaluation. Finally, Strevens proposes a test to determine which factors count as difference makers, called the eliminative procedure. This test can easily be adapted to the purposes of target system evaluation. More specifically, as I will show in this section, the two steps of the test correspond to and preserve the important aspects of partitioning and omission respectively.

However, I stress that Strevens's account is an analogue for target system evaluation. That is, we can apply Strevens's framework for determining what is relevant for a scientific explanation to determining what parts of the world are relevant for a particular scientific purpose. The fact that his account focuses on explanation rather than other purposes of modelling (such as prediction or confirmation) does not mean that target systems can only be evaluated in terms of the context of explanation. The

\footnotetext{
${ }^{6}$ In philosophy of language, relevance constitutes an important aspect of the pragmatics of communication. For example, Grice argued that conversations should be understood as cooperative enterprises. Bringing relevant information to the conversation is a maxim that speakers ought to follow in order for a conversation to take place (Davis 2014).
} 
account works as an analogue because explanations, like target systems, can only be evaluated given a particular context. In the case of target systems, the context does not have to be using a model for the purposes of explanation. The same method of evaluation can be used, even if the context is using a model for prediction, confirmation etc. In fact, my account of aptness can show why a target system that is apt for the purpose of explanation is inapt for the purpose of prediction. For example, if scientists wished to predict the growth of the VIM with the introduction of a new predator, then the target system described here would be inapt, as it assumes that predation is irrelevant. While we know that predation from the existing predators is not relevant, a new predator might have an important effect on the VIM growth.

According to Strevens $(2004,2008)$ the key for explaining an event is to pick out, from the complex causal network, those factors which made a difference to whether or not the event in question occurred. He argues that a key issue for causal accounts of explanation is their (in)ability to determine which causal factors are actually relevant for a particular explanation, and which have a negligible degree of influence (Strevens 2008). For example, an event such as the death of Rasputin, could have been caused by a number of factors (he was famously poisoned, shot (twice) and finally tied up and drowned). A good explanation of the event will allow us to identify drowning as the difference-maker, i.e. the factor that ultimately caused Rasputin's death.

More specifically, explaining an event $E$ begins by examining the causal network in which $E$ is embedded, and picking out a part of that web that was sufficient to cause $E$. By 'part' Strevens means a set of actual initial conditions, and laws that could, in itself cause $E$. Yet Strevens also points out that in order to determine this causal network, we must first determine exactly what counts as the event $E$. In other words, we need to decide how finely grained we want our event to be specified. For instance, the answer to the question 'why did Rasputin die' will elicit an explanation with fewer causal details than the explanation elicited by the question 'why did Rasputin die in exactly the way he did?' (Strevens 2004, p. 159). The second step is to construct a representation of this set of actual causes, which is called the veridical causal model (p. 162). It is a veridical model, because the conditions are actual causal influences on the event. In the model, relations of causal production are represented through relations of logical entailment. In other words, Strevens uses the notion of logical entailment to represent a situation in the real world, where a set of initial conditions produce an event in virtue of laws (p. 163).

In the case of target systems, we need to determine the usefulness of a partition, so as to have the appropriate context with which to determine the relevance of the parts and properties that are in the target system. It is standard in scientific practice to have a set hypothesis, which is often phrased as a question. This question can be used to determine how we should partition the domain. For example, in the case of the VIM, the question was 'why is the VIM population dropping, even though there are abundant resources?'. This question determines the context for relevance, for example that marmots, resources and marmot demographics should somehow feature in the target system. Other aspects of the domain are not so clearly useful. For example, do we actually need to distinguish between male and female marmots? At first glance, this may seem unnecessarily fine-grained. However, as we have seen, 
birth rates are a necessary aspect of determining $r$ for the logistic growth model, hence the partition should be this fine-grained. In contrast, there is no need for an even finer-grained partition, e.g. at the level of marmot alleles. While they are obviously there as parts of marmots, the partition does not need to identify them as separate parts.

In the VIM case, the appropriate level of partition was quite obvious, however, there are other cases where this is not so. For example, there is a debate in invasive species research about the appropriate way to partition domains. On one side are those who believe domains should be partitioned into individual organisms and their properties, as invasions occur because some traits give invaders a competitive advantage (Jarchow and Cook 2009; Richardson and Rejmánek 2004). On the other side, are those who believe that domains should be partitioned in terms of whole communities, because community-level traits are the difference-makers in terms of whether an invasion succeeds or not (Kennedy et al. 2002; Levine and D'Antonio 1999). Both sides have used models to successfully explain and predict particular invasion events (see discussion in Blackburn et al. 2011; Hayes and Barry 2007).

Still, looking more closely at the target systems shows that the finer grained partition is more likely to yield successful results. The reason is that the finer grained partition allows scientists to include a greater variety of causal factors in their target systems, while the coarser grained partitions are more limited. For example, with a finer grained partition, scientists can include traits that give invaders a competitive advantage and traits that place native organisms at a disadvantage. In contrast, the coarser-grained partition does not allow scientists to include the traits that confer the competitive advantage to the invaders. The point here is not that the coarser grained partitions are never successful, but that tend to be less useful for determining the outcome of an invasion.

Returning to Strevens's account, the third step is to eliminate from the causal model any factors that are not necessary in the causal production of $E$, i.e. that do not entail $E$. What remains is the set of initial conditions and laws that made a difference to the occurrence of $E$ - the explanatory kernel of $E$ (2004, p. 163). The causal network includes the drowning, the shooting and the poisoning, but also a number of other factors which have some causal influence on $E$, such as "the length of Rasputin's beard, the day's pollen count, the gravitational influence of Mars” (p. 158).

It is easy to see why drowning counts as a difference maker. 'Being thrown in the river' is part of the causal network that entails $E$, therefore is part of the veridical model. Also contained is a law which states that 'people thrown in the river under certain conditions die', and the conditions for which the law holds. Therefore, we cannot remove the being thrown into the river without invalidating the entailment. This means that being thrown into the river is a difference maker of $E$. Contrast this with the explanation of Rasputin's death by poisoning. To do so we would construct a causal model which contained the proposition that Rasputin was poisoned, together with a law which stated that a person poisoned under a set of conditions, will die. The problem is that in Rasputin's case, these conditions did not hold. We now have two options. Either we bite the bullet and state the conditions were present or we eliminate the conditions from the model. However, if we choose the first option, we end up with a causal model which is not veridical, as it asserts something 
which not the case. On the other hand, if we eliminate the conditions from the causal model, then we are left with a model which does not entail $E$. This is because, as Rasputin showed, simply being given a certain poison does always result in death. Thus, the poisoning cannot be considered a difference-maker for Rasputin's death.

We can apply a similar analysis to test which causal factors should be part of the target system. The parts of the domain that give rise to phenomenon are the equivalent of the veridical causal model. This includes the marmots and their properties, along with the resources of the environment and other causal factors, such as predators. The marmots and their demographic properties are clearly relevant, because if we omit them, then the Allee effect does not occur. The resources of the environment are also relevant, even though they are not the cause of this particular type of decline. This is because resources are important for many aspects of VIM life and removing them or changing them would change the growth rate of the VIM (probably by accelerating their decline). In order to have this growth rate, resources are necessary aspects of the target system.

The equivalent to the poison, in this case, are the predators. Here, the scientists knew that predators could cause the VIM population to drop, hence they considered them. However, they determined that changing the amount of predators (within a certain range) would not have changed the behaviour of the system. Thus, predators were not a relevant factor and could be excluded from the target. How do scientists actually determine whether a factor is relevant? They (with one or two exceptions) ${ }^{7}$ do not go around culling or fumigating parts of the world to test if a factor was indeed relevant. Instead, they make inferences about the performance of a particular system based on background knowledge and data and knowledge of similar systems. In this case, the scientists could eliminate predators from the target because they knew that predation rates were constant before and after the drop in the VIM population.

Of course, sometimes this information is not available. Then, scientists will have to conduct an experiment to determine whether something really is a relevant causal factor, or run different versions of a model, with and without the causal factor. In fact, there are some cases where scientists make mistakes, such as believing a factor to be irrelevant when it is, in fact, relevant. In this case, the sociality of the VIM could have been such a factor. Yet because of the preliminary work conducted to specify the target system, the scientists were able to identify it as a relevant factor. That is, they were aware of the fact that the VIM, unlike other marmots, were highly social, so when the simple logistic growth model did not line up with the VIM population decline, they considered it as a relevant factor. This kind of mistake is to be expected in science, especially when a discipline is young or when a new phenomenon is being investigated. Nonetheless, I believe that paying closer attention to the target system can help to mitigate the frequency and effect of these mistakes. I will illustrate with an example from plant ecology.

\footnotetext{
7 See for example Simberloff and Wilson's (1969) fumigation experiments on islands in Florida, in order to study migration, which was instrumental in the establishment of the theory of biogeography.
} 
For many years, various models (e.g. of competition, invasion, drought sensitivity) yielded erratic and surprising results when applied to plant populations (Berger et al. 2008; Phillips et al. 2016; Suding et al. 2013). Plant ecologists were unable to explain why this was the case, until they realised that when it comes to plants, what happens below the ground is much more important than what happens above it (Phillips et al. 2016). They discovered that many models suffer from a 'surface bias', as they do not include below-ground factors. Including these factors in the models increases their performance (i.e. yields more accurate explanations and predictions). According to Phillips et al., the scientific community was aware of the existence of these below-ground factors, yet they did not believe they were important enough to include in the models. When the scientists re-examined the systems, looking for factors that could be relevant, i.e. re-examining their target systems, they found the missing factors and improved their models. In fact, this realisation has resulted in the establishment of a new field (plant-soil feedback), which has revolutionised our understanding of plant competition and invasiveness (van der Putten 2013).

To sum up, scientists can test the aptness of their target by determining whether their partitions are useful for their investigation and whether the have correctly identified which parts and properties should be omitted from the system. These tests are based on background knowledge and data and is far from foolproof. Nonetheless, paying attention to the aptness of a target at an early stage of an investigation can increase the likelihood that the investigation's results are successful.

\section{Loose ends: general and hypothetical targets}

Even if we agree that target systems can be real-world systems, does this mean that all target systems are real world systems? In the literature, there are two interesting cases with alternative conceptions of target systems: targets of general models and hypothetical targets. I will examine each of these cases in turn, arguing that they are legitimate ways to understand target systems, but conceptually dependent on realworld targets.

\section{Targets of general models}

In his classifications of models and modelling, Weisberg (2013) argues that in some cases scientists construct general models, because they are not interested in particular instances of phenomena, but in classes of phenomena. These models are often very simple, in the sense that they aim to capture only the basic dynamics of a system and include very little detail. For example, he argues that a generalized model of sexual reproduction is not meant to uncover facts about sexual reproduction in particular populations, but to understand larger issues such as the relative merits of sexual reproduction when compared to asexual reproduction. Weisberg identifies two distinct functions for general models. The first is their use in minimalist idealization, when scientists construct models that capture the core causal factors a phenomenon that are common across many different manifestations of that phenomenon, but 
leave out the details that are particular to various manifestations (p. 119). The second is the generation of how-possibly explanations, explanations of how a phenomenon could come about, rather than why it did come about e.g., "what is a possible reason for sexual reproduction when asexual reproduction is less costly?" (p. 118).

According to Weisberg (2013), the target systems of general models are themselves general. They are abstractions over particular target systems that lie at the intersection of a number of specific targets and have the features common to all targets that the model can represent. For example, in the case of sexual reproduction, the generalized target is meant to have the set of properties are shared by all sexually reproducing species. Once the relevant features shared by all specific targets are identified, the scientist can abstract further by leaving out all the specifics of each case and focusing only on those generalized properties. This could spell trouble for my account, as general, abstract targets cannot be said to be real parts of the world.

There are two ways to respond to this point. The first is that on Weisberg's account, abstract targets are causally and ontologically dependent on particular targets. Scientists construct the generalized target by identifying the features that all the particular targets share, and then abstracting over them. ${ }^{8}$ Thus, if we allow for the existence of abstract targets, we must also allow for the existence of particular targets. This means that the mere existence of abstract targets is fully compatible with my account of particular, real world targets.

Second, Weisberg seems to imply that scientists using general models are not interested in particular manifestations of a phenomenon in various real-world populations. For example, he argues that the point of studying sex in a general manner is not to discover facts about sexual reproduction in particular populations, but to understand larger issues such as the relative merits of sexual reproduction when compared to asexual reproduction. Thus, "generalized model of sexual reproduction isn't supposed to be about kangaroo sex or fungi sex, but about sex itself." (p. 115). It seems that Weisberg is only considering one of the two functions he previously identified, i.e. how possibly explanations. In fact, many general models are used to learn about real world populations, many real-world populations. For example, the model used for the VIM is, by all accounts, a general model of population growth, which has been applied to the particular target of the VIM. Of course, this is not the only target of this general model. In fact, one could argue that what makes the logistic growth model general is not that it informs as about growth in general, but that it has many particular targets; it informs us about Vancouver Island marmot growth and bacteria growth and Arabidopsis growth and so on. In these cases,

\footnotetext{
8 Weisberg also states that there are more complex cases where the model is less abstract than the target (e.g. individual-based models). In these cases, he argues that the scientist must restrict the model's scope so that it conforms to the actual target. For example, if a scientist wished to construct an agent-based model of sexual reproduction she would need to add concrete properties such as the life-cycle, spatial distributions and fitness of individual organisms, which are not part of the abstract target. It is unclear how, given this necessary scope restriction, it would be possible to ever construct an agent-based model of sexual reproduction. However, the reason for the mismatch is the reliance on the previously identified 'abstract target'. It seems much more plausible to allow the scientist to apply the (less) abstract model to the particular real-world target she is interested in.
} 
the generalised target is simply shorthand for referring to a family of actual targets which share common properties. Thus, the only reason that general targets are useful is because they can be reduced to actual targets.

Still, Weisberg is right that there are some cases where scientists use general models to learn about phenomena in general. Even if we allow that there exist both real-world particular targets and abstract targets for each general model, we might want to determine which should take precedence as that which the model is about. A different way of putting the point is, should we be pluralists about the nature of target systems or are there reasons to favour one or the other type of target? I will address this issue in section "Pluralism about target systems?", after examining the possibility of hypothetical target systems.

\section{Targets of hypothetical models}

Weisberg's classification includes a second case of potentially non-real targets, those represented by hypothetical models. These are models that, by definition, do not represent particular systems in the world, as those systems do not or cannot exist (e.g. models of perpetual motion machines or genetics models with multiple sexes). ${ }^{9}$ Instead, they are thought to represent hypothetical systems or 'possibilities'. Take, for example, a model of a simple harmonic oscillator. The equations of the model describe a system that experiences a restoring force when displaced from its original position, and this force is proportional to its displacement. This model also describes the movement of pendula when they do not have any friction to dampen the oscillatory movement. Of course, there is no such thing as a pendulum in the world that does not experience any friction; it is probably impossible for such a pendulum to exist. What is the target system of the model in this case?

The answer depends on the model's intended use. If the scientists wish to learn about a hypothetical system, then their target will be hypothetical. Yet, the mere existence of hypothetical targets does not negate the existence of real-world particular targets. It is more probable that scientists will apply the model to the world, in order to learn about actual pendula (such as Ron Giere's grandfather clock). In cases like these it is quite obvious that the model's target is a real-world system (here the actual pendulum in Ron Giere's grandfather clock). The fact that the model does not take into account friction constitutes an idealization, thus the model is an idealized (and strictly speaking inaccurate) representation of the clock. This is no different to pretending that a population is infinitely large, smoothing out the surface of the sun for Newtonian planetary motion, or even merely changing the amount of friction in the model. Whether or not this is a good model for a particular purpose, depends on the goal of the scientists, and cannot be determined here (it is an empirical question). The point is, that the model represents the clock, not a hypothetical pendulum.

\footnotetext{
${ }^{9}$ I should note that hypothetical models should not be confused with targetless models. The latter are intended not to be about any type of target-real, imaginary or otherwise-and hence are not relevant for this discussion.
} 
Of course, just like in the case of general models, one could argue that the model represents the clock in addition to it representing a hypothetical pendulum. I turn to this issue next.

\section{Pluralism about target systems?}

Even if we agree that some or even most target systems are real parts of the world, we might still wish to adopt a pluralistic attitude towards the ontological status of target systems. Weisberg's motivation behind the claim that general and hypothetical models have corresponding general and hypothetical targets is the intentions of the scientists that use them. In both cases, he maintains that scientists are not interested in real, particular systems, but in abstract or hypothetical systems. In the previous two sections I showed that this claim is overstated, as scientists utilizing general and hypothetical models are often interested in learning about real, particular systems.

Nonetheless, it is a much stronger claim to maintain that scientists are never interested in learning about general or hypothetical systems, and one which I have no reason to make. It suffices, for my purposes, to show that a significant proportion of target systems are real particular systems. Their reality stems from them being parts of the world, and they come about because of how scientists apply their models to the world. Yet this does not mean that scientists are always interested in learning about parts of the world, or indeed applying every single instance of a model to a part of the world. In these cases, I agree that their models do not target parts of the world.

The question is whether we should call these systems target systems, or whether we should restrict the term to the systems that are real parts of the world. While I think that this type of pluralism can be confusing, I am not opposed to it in principle. It is acceptable for a pluralist to denote all these systems target systems, provided that there is sufficient attention paid to the real-world target systems, and their value for scientific investigations. If, on the other hand, the call for pluralism stems from the notion that identifying the real-world target is uninteresting or unimportant, despite claims that the model is intended to be informative about a part of the world, then this is much more problematic.

\section{Conclusion}

Target systems are best understood as concrete parts of the world. There are cases where it may help to conceptualise a target as an abstract or hypothetical system, yet these conceptions are dependent on the conception of targets as parts of the world. Target systems have a place in discussions of scientific modelling, because target system specification is a crucial aspect of scientific practice. Even though target systems are parts of the world, they can more or less apt with respect to a particular purpose. Moreover, target system specification takes up a lot of time, effort and resources but can also be extremely valuable, as it allows scientists to understand the causes of model failure (i.e. failure to predict or explain a phenomenon). Paying 
close attention to a particular target system can also help scientists to choose the right model or construct it in such a way as to minimise the risk of failure.

\section{Appendix}

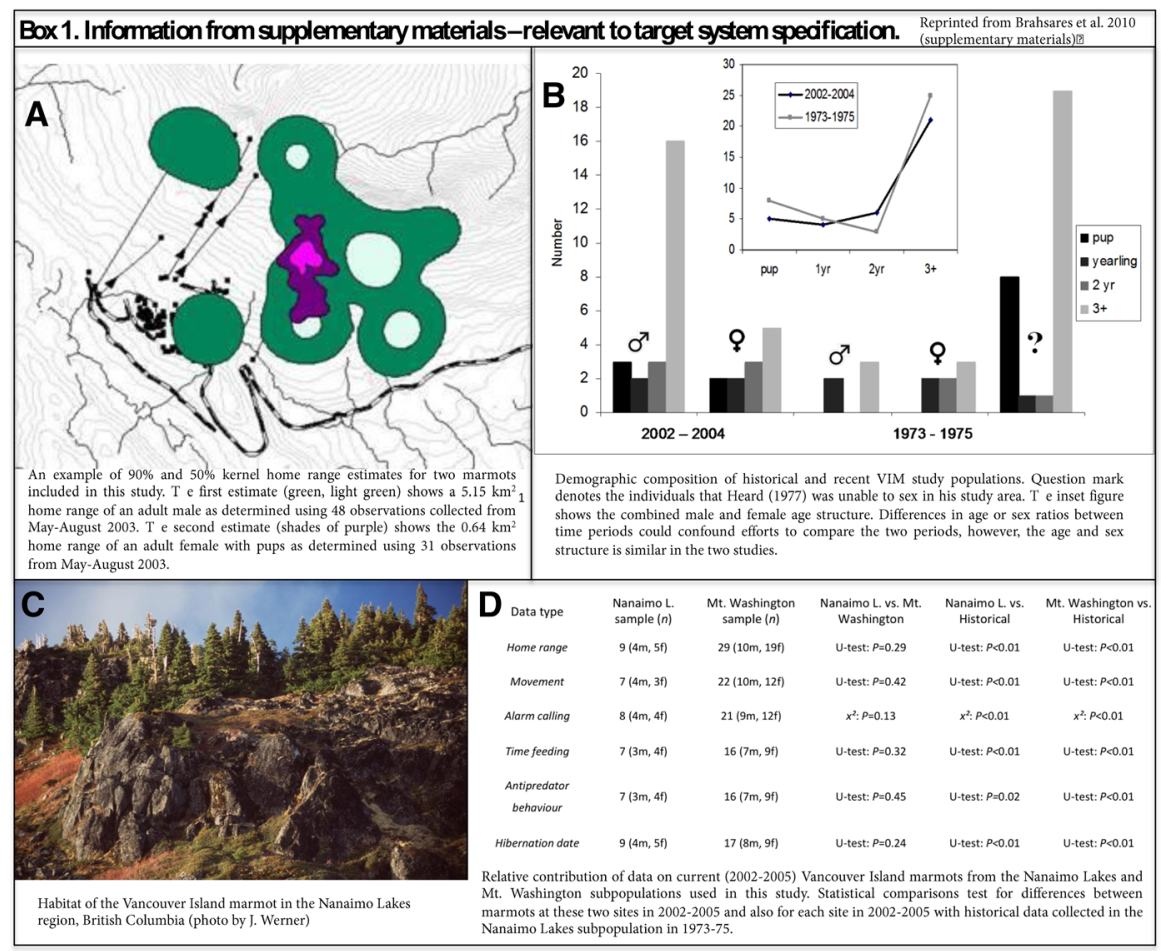

Acknowledgements Open access funding provided by University of Helsinki including Helsinki University Central Hospital. I would like to thank the editor and two anonymous reviewers for helpful and constructive comments on the paper. I would also like to thank the philosophers at the University of Pennsylvania, especially Michael Weisberg, Cristina Bicchieri, Liz Camp, Dan Singer, Zoltan Domotor and Scott Weinstein for discussions that helped these ideas take form and for their support while these ideas were developing.

Open Access This article is licensed under a Creative Commons Attribution 4.0 International License, which permits use, sharing, adaptation, distribution and reproduction in any medium or format, as long as you give appropriate credit to the original author(s) and the source, provide a link to the Creative Commons licence, and indicate if changes were made. The images or other third party material in this article are included in the article's Creative Commons licence, unless indicated otherwise in a credit line to the material. If material is not included in the article's Creative Commons licence and your intended use is not permitted by statutory regulation or exceeds the permitted use, you will need to obtain permission 
directly from the copyright holder. To view a copy of this licence, visit http://creativecommons.org/licen ses/by/4.0/.

\section{References}

Berger U, Piou C, Schiffers K, Grimm V (2008) Competition among plants: concepts, individualbased modelling approaches, and a proposal for a future research strategy. Perspect Plant Ecol Evol Syst 9(3-4):121-135

Blackburn TM, Pyšek P, Bacher S, Carlton JT, Duncan RP, Jarošík V et al (2011) A proposed unified framework for biological invasions. Trends Ecol Evol 26(7):333-339

Brashares JS, Werner JR, Sinclair ARE (2010) Social "meltdown" in the demise of an island endemic: Allee effects and the Vancouver Island marmot. J Anim Ecol 79:965-973

Cartwright N (1989) Nature's capacities and their measurement. Oxford University Press, Oxford

Courchamp F, Clutton-Brock T, Grenfell B (1999) Inverse density dependence and the Allee effect. Trends Ecol Evol 14(10):405-410

Davis W (2014) Implicature. In: Zalta EN (ed) The Stanford encyclopedia of philosophy (Fall 2014 edition). https://plato.stanford.edu/archives/fall2014/entries/implicatu. Accessed 5 Oct 2015

Evans $\mathrm{M}$ et al (2013) Do simple models lead to generality in ecology? TREE 28(10):578-583

Frigg R (2009) Models and fiction. Synthese 172(2):251-268

Giere R (2004) How models are used to represent reality. Philos Sci (Proc) 71(5):742-752

Godfrey-Smith P (2009) Abstractions, idealizations, and evolutionary biology. In: Barberousse A, Morange M, Pradeu T (eds) Mapping the future of biology, vol 266. Springer, Dordrecht, pp $47-56$

Hayes KR, Barry SC (2007) Are there any consistent predictors of invasion success? Biol Invasions 10(4):483-506

Humphreys P (1995) Abstract and concrete. Philos Phenomenol Res 55(1):157-161

Jarchow ME, Cook BJ (2009) Allelopathy as a mechanism for the invasion of Typha angustifolia. Plant Ecol 204(1):113-124

Jones MR (2005) Idealization and abstraction: a framework. In: Jones MR, Cartwright N (eds) Idealization XII: correcting the model. Idealization and abstraction in the sciences. Rodopi, Amsterdam, pp 173-217

Kennedy TA, Naeem S, Howe KM, Knops JMH, Tilman D, Reich P (2002) Biodiversity as a barrier to ecological invasion. Nature 417(6889):636-638

Kingsland S (1995) Modeling nature. University of Chicago Press, Chicago

Knuuttila T (2005) Models, representation, and mediation. Philos Sci 72(5):1260-1271

Levine JM, D’Antonio CM (1999) Elton revisited: a review of evidence linking diversity and invasibility. Oikos 87(1):15

Levy A (2018) Idealization and abstraction: refining the distinction. Synthese 13(1):1-18

Mäki U (2009) MISSing the world: models as isolations, representations, and credible worlds. Erkenntnis 70:29-43

Matthewson J, Weisberg M (2009) The structure of tradeoffs in model building. Synthese 170(1):169-190

May R (2001) Stability and complexity in model ecosystems. Princeton University Press, Princeton

Odenbaugh J (2007) Seeing the forest and the trees: realism about communities and ecosystems. Philos Sci 74(5):628-641

Perretti C, Munch S (2013) Model-free forecasting outperforms the correct mechanistic model for simulated and experimental data. PNAS 110(13):5253-5257

Peschard I (2010) Target systems, phenomena and the problem of relevance. Mod Sch 87(3-4):267-284

Phillips RP, Ibanez I, D’Orangeville L (2016) A belowground perspective on the drought sensitivity of forests: towards improved understanding and simulation. For Ecol Manag 380:309-320

van der Putten WH et al (2013) Plant-soil feedbacks: the past the present and future challenges. J Ecol 101(2):265-276

Richardson DM, Rejmánek M (2004) Conifers as invasive aliens: a global survey and predictive framework. Divers Distrib 10(5-6):321-331 
Ricklefs RE, Miller GL (2000) Ecology. Macmillan, New York

Sackett TE, Classen AT, Sanders NJ (2010) Linking soil food web structure to above- and belowground ecosystem processes: a meta-analysis. Oikos 119(12):1984-1992

Shrader-Frechette K, McCoy E (1993) Method in ecology. Cambridge University Press, Cambridge

Simberloff D, Wilson E (1969) Experimental zoogeography of Islands: the colonization of empty islands. Ecology 50(2):278-296

Strevens M (2004) The causal and unification approaches to explanation unified-causally. Noûs 38(1):154-176

Strevens M (2008) Depth. Harvard University Press, Cambridge

Suarez M (2003) Scientific representation: against isomorphism and similarity. Int Stud Philos Sci 17(3):225-244

Suding KN, Harpole WS, Fukami T et al (2013) Consequences of plant-soil feedbacks in invasion. J Ecol 101:298-308

Travis J et al (2014) Integrating the invisible fabric of nature into fisheries management. PNAS 111:2 Vancouver Island Marmot Recovery Team (2008) Recovery strategy for the vancouver island marmot (Marmota vancouverensis) in British Columbia. Prepared for the B.C, Ministry of Environment, Victoria, BC

Weisberg M (2013) Simulation and similarity. OUP, Oxford

Publisher's Note Springer Nature remains neutral with regard to jurisdictional claims in published maps and institutional affiliations. 